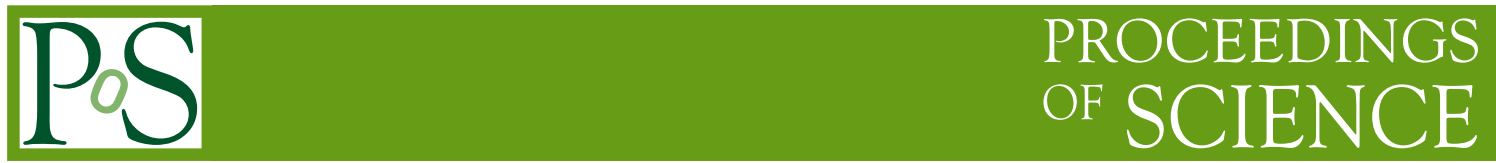

\title{
New results on the Be-8 anomaly
}

\author{
A.J. Krasznahorkay ${ }^{1 *}$ M. Csatlós ${ }^{1}$, L. Csige ${ }^{1}$, J. Gulyás ${ }^{1}$, M. Hunyadi ${ }^{1}$, T.J. Ketel ${ }^{2}$, \\ A. Krasznahorkay ${ }^{3}$, I. Kuti ${ }^{1}$, Á. Nagy ${ }^{1}$, B.M. Nyakó ${ }^{1}$, N. Sas ${ }^{1}$, J. Timárr ${ }^{1}$ I. Vajda ${ }^{1}$ \\ ${ }^{1}$ Institute for Nuclear Research, Hungarian Academy of Sciences, MTA Atomki \\ ${ }^{2}$ Nikhef Nat. Inst. for Subatomic Phys., Science Park 105, 1098 XG Amsterdam, The Netherlands \\ ${ }^{3}$ CERN,European Organization for Nuclear Research, Geneva, Switzerland \\ E-mail: kraszna@atomki.hu
}

Recently, we measured the $\mathrm{e}^{+} \mathrm{e}^{-}$angular correlations in internal pair creation (IPC) for the M1 transitions depopulating the 17.6 and $18.15 \mathrm{MeV}$ states in ${ }^{8} \mathrm{Be}$, and observed a peak-like deviation from the predicted IPC for the $18.15 \mathrm{MeV}$ transition, but not for the $17.6 \mathrm{MeV}$ one. To the best of our knowledge no nuclear physics related description of such deviation can be made. The deviation between the experimental and theoretical angular correlations is significant and can be described by assuming the creation and subsequent decay of a boson with mass $m_{0} c^{2}=16.70 \pm 0.35$ (stat) \pm 0.5 (sys) MeV. The branching ratio of the $\mathrm{e}^{+} \mathrm{e}^{-}$decay of such a boson to the $\gamma$ decay of the $18.15 \mathrm{MeV}$ level of ${ }^{8} \mathrm{Be}$ is found to be $5.8 \times 10^{-6}$ for the best fit. The data can be explained by a $17.6 \mathrm{MeV}$ vector gauge boson $\mathrm{X}$ that is produced in the decay of the excited state to the ground state, and then decays to $\mathrm{e}^{+} \mathrm{e}^{-}$pairs.

In the present work we re-investigated the $\mathrm{e}^{+} \mathrm{e}^{-}$pair correlation in the $17.6 \mathrm{MeV}$ transition of

${ }^{8} \mathrm{Be}$ in which some smaller deviation was also expected. The branching ratio of the $e^{+} e^{-}$decay of such a boson to the $\gamma$ decay was found to be $(2 \pm 2) \times 10^{-6}$.

55th International Winter Meeting on Nuclear Physics

23-27 January, 2017

Bormio, Italy

${ }^{*}$ Speaker. 


\section{Introduction}

Dark matter is currently one of the greatest unsolved mysteries in physics. The leading darkmatter candidates are Weakly Interacting Massive Particles (WIMPs), axions, dark photons, etc. predicted by some physics theories but never detected. WIMPs are expected to have a mass of 10 to 100 times heavier than a neutron. There have been dozens of big experiments that have searched for dark matter and found no sign of it yet.

In various dark matter theories, a kind of popular model includes a light new boson $\mathrm{X}$ which mediates between the Standard Model and a dark sector, e.g., Ref. [1, 2].

Very recently, we have observed an anomaly in the nuclear decay of ${ }^{8} \mathrm{Be}$. This could be a first hint for a $17 \mathrm{MeV} \mathrm{X}$-boson signal [3]. The relevant level scheme of ${ }^{8} \mathrm{Be}$ is shown in Fig. 1.

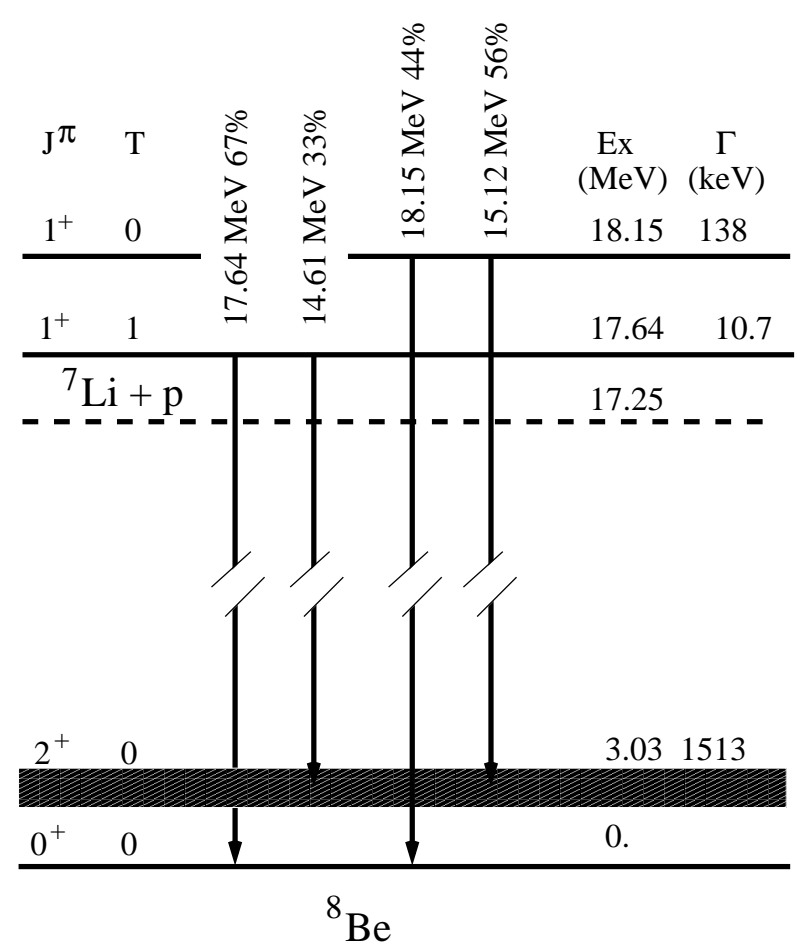

Figure 1: The most relevant 8Be states and their spin-parities $J^{\pi}$, isospins $T$, excitation energies Ex, and decay widths $\Gamma$ from Ref. [23]. Decays of the ${ }^{8}$ Be $18.15 \mathrm{MeV}$ state to the ground state of ${ }^{8}$ Be exhibit anomalous internal pair creation.

The ${ }^{7} \mathrm{Li}(\mathrm{p}, \gamma)^{8} \mathrm{Be}$ reaction was used at the $\mathrm{E}_{p}=0.441 \mathrm{MeV}$ and $\mathrm{E}_{p}=1.03 \mathrm{MeV}$ resonances to populate the excited states in ${ }^{8} \mathrm{Be}$ selectively and the differential internal pair conversion coefficients were studied for the $17.6-\mathrm{MeV}$, and $18.15-\mathrm{MeV}, J^{\pi}=1^{+} \rightarrow 0^{+}, \mathrm{M} 1$ transitions in ${ }^{8} \mathrm{Be}$. Significant peak-like enhancement of the internal pair creation was observed at large angles in the angular correlation of the $18.15 \mathrm{MeV}$ transition, but not in the $17.6 \mathrm{MeV}$ one [3]. This observation was interpreted as the creation and the subsequent decay of a neutral boson with mass $m_{0} c^{2}=16.70 \pm 0.35$ (stat) \pm 0.5 (sys) MeV. The branching ratio of the $e^{+} e^{-}$decay of such a boson to the $\gamma$ decay of the $18.15 \mathrm{MeV}$ level of ${ }^{8} \mathrm{Be}$ is found to be $5.8 \times 10^{-6}$ for the best fit [3]. 
Zhang, Gerald and Miller discussed in detail if nuclear physics could explain the anomaly observed in the internal pair production in the ${ }^{8} \mathrm{Be}$ nucleus. They have improved the previous nuclear physics model for the $\mathrm{e}^{+} \mathrm{e}$ production in the current experimental context, by including the interferences between E1, E2, and M1 multipoles and two different angular dependencies in the modelings, and introducing important constraints from the photon production measurements. These improvements, should be included in extracting new particle properties from the experiment of this type, but can not explain the observed anomaly within nuclear physics [4].

A theoretical group lead by J. Feng [5, 6] studied our data as well as all other previous experiments in this area and showed that the evidence strongly disfavors dark photons. They proposed a new theory, however, that synthesizes all existing data and determined that the discovery could indicate a fifth fundamental force [5]. They explained our experimental data by a $17.6 \mathrm{MeV}$ vector gauge boson $\mathrm{X}$ that is produced in the decay of the excited state to the ground state, and then decays to $\mathrm{e}^{+} \mathrm{e}^{-}$pairs. If confirmed by further experiments, this discovery of a possible fifth force would completely change our understanding of the universe.

The protophobic $17 \mathrm{MeV}$ gauge boson can mediate isovector transitions, so there is no dynamical suppression of this decay. However, its mass is near the $17.64 \mathrm{MeV}$ threshold, so that the decay is kinematically suppressed noted Feng et al. [5]. They calculated the suppression factor for particle mass of $17 \mathrm{MeV}$ and $17.4 \mathrm{MeV}$ and obtained values of 2.3 and 5.2, respectively. In spite of that suppression, it would be important to see the anomaly also in the $17.64 \mathrm{MeV}$ transition, since it is a much cleaner case without having any interference effect, so we decided to repeat that experiment with better conditions than before [3].

In the present paper we are reporting on a new experiment aiming at studying the $17.6 \mathrm{MeV}$ transition with reduced systematic uncertainties.

As we reported previously [7, 8], our experimental setup has been moved to a new accelerator laboratory and has also been improved. We repeated the previous experiments and observed similar deviations from the internal pair creation at large angles in the angular correlations for the 18.15 transition, like previously [3], and we observed some smaller deviation also for the $17.6 \mathrm{MeV}$ transition as was predicted by Feng et al., [5], but which we did not see before [3].

In the present experiment the detector alignments, especially for the DSSD detectors were made more precise. The precision of their positioning is now well below $1 \mathrm{~mm}$. The statistics were also improved. The number of the $\mathrm{e}^{+} \mathrm{e}^{-}$pairs measured from the decay of the $17.6 \mathrm{MeV}$ excited state has been increased by about a factor of 3 to 103 thousand true coincidence events compared to our previous results $[7,8]$.

The possible relation of the $\mathrm{X}$ boson to the dark matter problem as well as the fact that it might explain the $(\mathrm{g}-2)_{\mu}$ puzzle $[11,12]$, triggered an enormous theoretical and experimental interest in the particle and hadron physics community. In the following section we will just mention a few relevant articles.

\section{Reflections to our article on the ${ }^{8}$ Be anomaly}

Ellwanger and Moretti [9] made a possible explanation of the electron positron anomaly at 17 $\mathrm{MeV}$ in ${ }^{8} \mathrm{Be}$ transitions through a light pseudo-scalar particle. Kozaczuk, Morrissey and Stroberg [10] investigated the production of the $\mathrm{X}(17)$ vector boson with primarily axial couplings to light 
quarks in nuclear transitions. The relevant matrix elements for the $8 \mathrm{Be}$ transitions were calculated using ab initio methods. They found that the emission of X(17) can account for the anomaly seen in the $18.15 \mathrm{MeV}$ isoscalar transition together with the absence of a significant anomaly in the corresponding $17.6 \mathrm{MeV}$ isovector transition.

Kitahara and Yamamoto showed that such a new particle could mediate between the Standard Model and a dark sector, which includes the dark matter [2]. Chiang and Tseng suggested to probe the $\mathrm{X}(17)$ particle using rare leptonic kaon and pion decays. There are several analysis on how to further test the model in Ref. [5] using low-energy physical processes, as well as proposals of alternative models for the ${ }^{8} \mathrm{Be}$ anomaly [13]. Most recently Liang, Chen, and Qiao found that the newly observed boson X(16.7) may be the solution of both the NuTeV anomaly and the (g-2) $\mu$ puzzle.

The ATLAS Collaboration [15] presented results of a search for long-lived neutral particles decaying into collimated jets of light leptons and mesons, so-called "lepton-jets", using a sample of $3.4 \mathrm{fb}^{-1}$ of proton-proton collisions data at a center-of-mass energy of $\sqrt{s}=13 \mathrm{TeV}$ collected during 2015 with the ATLAS detector at the LHC. Assuming conventional production cross section to the dark sector of $5.0 \mathrm{pb}$ for a $800 \mathrm{GeV}$ heavy scalar boson, dark photon $\mathrm{c} \tau$ is excluded in the range $0.6 \mathrm{~mm} \leq c \tau \leq 63 \mathrm{~mm}$ for the Higgs $\rightarrow 2 \gamma \mathrm{d}+\mathrm{X}$ model and in the range $0.8 \mathrm{~mm} \leq c \tau \leq$ $186 \mathrm{~mm}$ for the Higgs $\rightarrow 4 \gamma \mathrm{d}+\mathrm{X}$ model. These exclusions does not effect X(17), with a deduced coupling to electrons in the range of $2 \times 10^{-4} \leq \varepsilon_{e} \leq 1.4 \times 10^{-3}$ which could explain the Be- 8 anomaly.

The DarkLight experiment [16] proposes to search for dark photon and the $\mathrm{X}(17)$ through complete reconstruction of the final states of electron-proton collisions. In order to accomplish this, the experiment requires a moderate-density target and a very high intensity, low energy electron beam.

Araki et al, [17] discussed the feasibility of detecting the gauge boson of the U(1) symmetry, which possesses a mass in the range between $\mathrm{MeV}$ and $\mathrm{GeV}$, at the Belle-II experiment. They have found that the Belle-II experiment with the designed luminosity can examine a part of the parameter region that evades the current experimental constraints and, at the same time, is favored by the observation of the muon anomalous magnetic moment.

Long-Bin Chen et al., [18] discussed, the production of the new $\mathrm{X}(17)$ boson in electronpositron collision, using BaBar, and the results are encouraging. The data collected at BESIII and $\mathrm{BaBar}$ turn out to be enough to perform a decisive analysis and hence give a definite answer to the existence of X(16.7). It turns out that at BESIII there should be about 82 - $4003 \mathrm{X}$ boson to be produced each year, and the numbers at B-factories are even higher, e.g. 113 - 5577 at BaBar with integrated luminosity of $514 \mathrm{fb}^{-1}$. With a precise measurement of the invariant-mass distribution of the final state $e^{+} e$ near the squared mass of $\mathrm{X}$ boson, and its decay length to further suppress the background, it is feasible to perform a decisive analysis for BESIII, BaBar, or SuperKEKB experiments and hence give a definite answer to the existence of $\mathrm{X}$ boson.

Marin Benito et al., [19] discussed the prospects for the search of $K_{S}^{0} \rightarrow \pi^{+} \pi^{-} e^{+} e^{-}$at LHCb. LHCb has proved to be very competitive in the search for such rare strange decays. The feasibility of observing such $\mathrm{K} 0$ decay at LHCb is studied using simulated and real data. During the Run I of LHC (2012), the yield of events expected per $\mathrm{fb}^{-1}$ of pp collisions at $\sqrt{s}=8 \mathrm{TeV}$ is found 
to be $\mathrm{N}($ Run1 $)=120_{-100}^{+280}$. A dedicated trigger selection has been developed for the 2016 datataking. A large signal yield, $\mathrm{N}($ Upgrade $)=(5 \pm 0.3) \times 10^{4} \mathrm{per}^{-1}$, is expected in the LHC upgrade phase. Pseudo experiments have been run to assess the feasibility of discovering evidence for the observation of the signal already in the Run I data-set.

Chian-Shu Chen et al., [20] studied the $17 \mathrm{MeV}$ anomaly in beryllium decays and U(1) portal to dark matter and concluded that it was possible to test the underlying U(1) portal model by the future $\mathrm{Si}$ and $\mathrm{Ge}$ detectors with $5 \mathrm{e}^{-}$threshold charges.

Rare leptonic kaon and pion decays $\mathrm{K}^{+}\left(\pi^{+}\right) \rightarrow \mu^{+} v_{\mu} e^{+} e^{-}$can also be used to probe a dark photon of mass $\mathrm{O}(10) \mathrm{MeV}$. Cheng-Wei Chiang [21] evaluated the reach of future experiments for the dark photon with vectorial couplings to the standard model fermions except for the neutrinos, and show that a great portion of the preferred $16.7-\mathrm{MeV}$ dark photon parameter space can be decisively probed.

The NA64/2 collaboration [22] proposed new measurements dedicated to the sensitive search for the $\mathrm{X} \rightarrow e^{+} e^{-}$decay of a new short-lived neutral boson, $\mathrm{X}$, with a mass $16.7 \mathrm{MeV}$ and coupling to electrons in the range $2 \times 10^{-4} \leq \varepsilon_{e} \leq 1.4 \times 10^{-3}$ which could explain an excess of $e^{+} e^{-}$pairs observed in the excited ${ }^{8} \mathrm{Be}^{*}$ nucleus transitions. If such $\mathrm{X}$ 's exist, they could be searched for in a light-shining-through-a-wall experiment with a high energy electron beam. The electron energy absorption in a calorimeter (WCAL) is accompanied by the emission of bremsstrahlung X's. A part of the primary beam energy is deposited in the WCAL, while the rest of the energy is transmitted by the X through the "WCAL wall" and deposited in another downstream calorimeter, ECAL, by the $e^{+} e^{-}$pair from the $\mathrm{X} \rightarrow e^{+} e^{-}$decay in flight. Thus, the X's could be observed by looking for an excess of events with the two shower signature generated by a single high energy electron in the WCAL and ECAL. A proposal to perform such an experiment to probe the still unexplored area of coupling strength $10^{-4} \leq \varepsilon_{e} \leq 10^{-3}$ and mass $\mathrm{m}_{X}=16.7 \mathrm{MeV}$ by using $100-150 \mathrm{GeV}$ electron beams from the CERN SPS is presented.

\section{Experiments}

To populate the $17.6 \mathrm{MeV} 1^{+}$states in ${ }^{8} \mathrm{Be}$ selectively, we used the ${ }^{7} \mathrm{Li}(\mathrm{p}, \gamma){ }^{8} \mathrm{Be}$ reaction at the $E_{p}=441 \mathrm{keV}$ resonance [23]. As we reported previously [7, 8], our experimental setup has been moved to a new accelerator laboratory and has also been improved. The multi-wire proportional counters were replaced with silicon DSSD detectors, as well as the complete electronics and data acquisition system was upgraded. The experiments were performed at the $2 \mathrm{MV}$ Tandetron accelerator in Debrecen. A proton beam with a typical current of $1.0 \mu \mathrm{A}$ impinged on $15 \mu \mathrm{g} / \mathrm{cm}^{2}$ thick $\mathrm{LiF}$ target evaporated on $10 \mu \mathrm{m}$ thick $\mathrm{Al}$ backing.

The $e^{+} e^{-}$pairs were detected by five plastic scintillator $\Delta E-E$ detector telescopes placed perpendicularly to the beam direction at azimuthal angles of $0^{\circ}, 60^{\circ}, 120^{\circ}, 180^{\circ}$ and $270^{\circ}$ [24]. The positions of the hits were registered by double sided silicon strip detectors having a strip widths of $3 \mathrm{~mm}$. The target strip foil was perpendicular to the beam direction. The telescope detectors were placed around the vacuum chamber made of a carbon fiber tube with a wall thickness of $1 \mathrm{~mm}$.

$\gamma$ rays were also detected for monitoring purposes. A $\varepsilon_{r e l}=100 \% \mathrm{HPGe}$ detector (measured at 1.33 MeV relative to that of a standard 3"-diameter, 3"-long $\mathrm{NaI}(\mathrm{Tl})$ scintillator) was used at 25 
$\mathrm{cm}$ from the target to detect the $17.6 \mathrm{MeV} \gamma$ rays in the ${ }^{7} \mathrm{Li}(\mathrm{p}, \gamma)^{8} \mathrm{Be}$ reaction. Typical $\gamma$-ray spectra measured at $\mathrm{E}_{p}=441 \mathrm{keV}$ is shown in Fig. 2 .

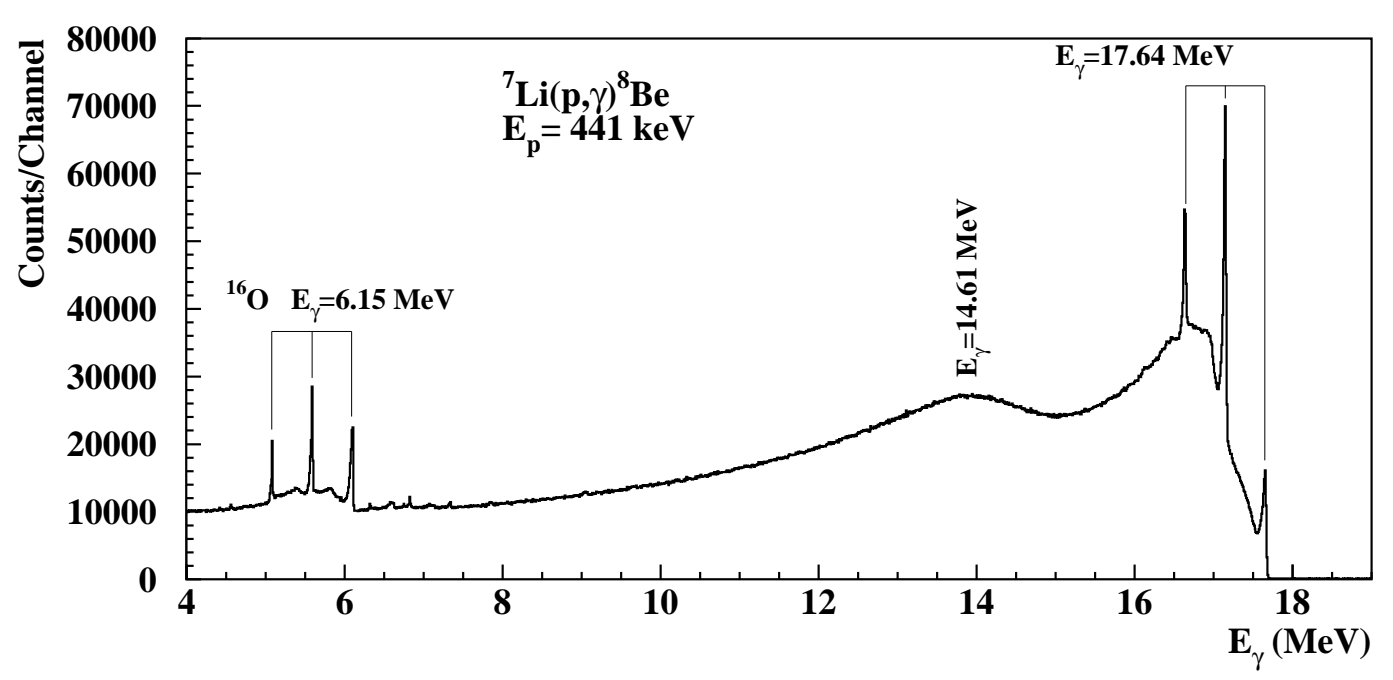

Figure 2: A typical $\gamma$-ray spectrum measured at $E_{p}=441 \mathrm{keV}$.

The $17.6\left(1^{+} \rightarrow 0^{+}\right)$photo-peak and their single and double escape peaks are clearly visible. The broad peaks at $14-15 \mathrm{MeV}$ correspond to transitions to the first excited $2^{+}$level at $\mathrm{E}_{x}=3.0$ $\mathrm{MeV}$, which has a width of $\Gamma=1.5 \mathrm{MeV}$ [23]. The branching ratios of $\gamma$-transition to the ground state and to the $2^{+}$state are about $70 \%$ and $30 \%$ from the $17.6 \mathrm{MeV} 1^{+}$state [23].

The excitation function of the reaction was also measured around the $441 \mathrm{keV}$ resonance in order to check the target thickness. The measured width of the resonance was found to be $15 \mathrm{keV}$ compared to the real width of $\Gamma=10.7 \mathrm{keV}$ taken from the literature [23]. From this we concluded that the energy loss of the protons in the target was sufficiently small $(8.7 \mathrm{keV})$. Therefore, we can be sure that the multipolarity of the transition is dominated by M1. The contribution of the direct capture process gives a small background with E1 multipolarity, but according to the excitation function measurements its contribution is less than $1 \%$ [25].

Figure 3 shows the total energy spectrum of $e^{+} e^{-}$pairs measured at the proton absorption resonance at $\mathrm{E}_{p}=441 \mathrm{keV}$. The strong $6.05-\mathrm{MeV}$ peak comes from the ${ }^{19} \mathrm{~F}(\mathrm{p}, \alpha){ }^{16} \mathrm{O}$ reaction followed by the $100 \%$ IPC transition $\left(0^{+} \rightarrow 0^{+}, \mathrm{E} 0\right)$. This transition was used for energy calibration of the spectrometer and also for checking its efficiency calibration, since the angular correlation of the $e^{+} e^{-}$pairs coming from this transition is well known.

The efficiency calibration of the telescopes was made by using the same dataset but with uncorrelated $e^{+} e^{-}$pairs coming from different events. We have learned that with the new setup we can not neglect the effect of the energy dependence of the efficiency any longer. It is mainly because the sensitive part of the DSSD detector $(50 \times 50 \mathrm{~mm} 2)$ is significantly larger compared to the size of the MWPC detectors $\left(30 \times 30 \mathrm{~mm}^{2}\right)$. In this way some events, which are detected close to 


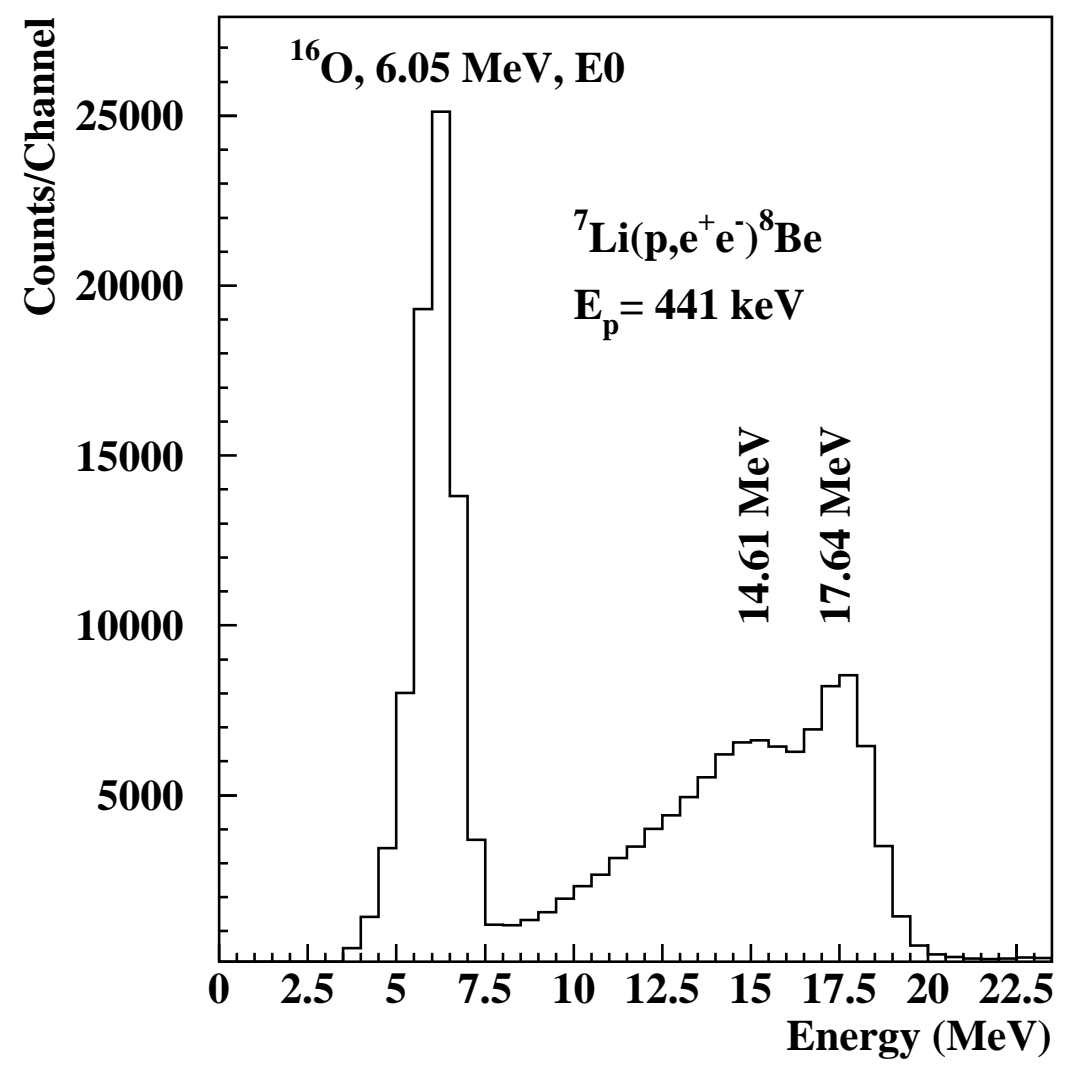

Figure 3: Total energy spectrum of $e^{+} e^{-}$pairs measured at $E_{p}=441 \mathrm{keV}$.

the surface of the plastic scintillators, may not be detected with full energy, as part of the radiation may escape from the scintillators.

We have made systematic Monte Carlo simulations for that effect, and were corrected the experimental results also for that.

Fig.4 shows our experimental results for the angular correlation of $e^{+} e^{-}$pairs measured at the proton absorption resonance at $\mathrm{E}_{p}=441 \mathrm{keV}$. In order to check the efficiency of the experimental setup we calculated the angular correlation also for the $6.05 \mathrm{MeV}$ E0 transition coming from ${ }^{16} \mathrm{O}$. It is shown in the upper curve of Fig.4 together with the simulated results for an E0 transition.

For the $17.6 \mathrm{MeV}$ transition we observed a slight deviation from the simulated pure M1 internal pair conversion correlation (IPCC) curve at large angles. A smoothly increasing difference could be originated from the direct (non-resonant) proton capture, the multipolarity of which is dominated by E1 [26], and it adds to the M1 decay of the resonance. The contribution of the direct capture depends on the target thickness if the energy loss of the beam in the target is comparable with the width of the resonance. The full simulated curve in Fig. 4 is obtained by adding a small E1 contribution (1.0\%) to the dominant M1 one, which describes the experimental data reasonably well, except the small peak-like deviation observed at about 150 degree. 


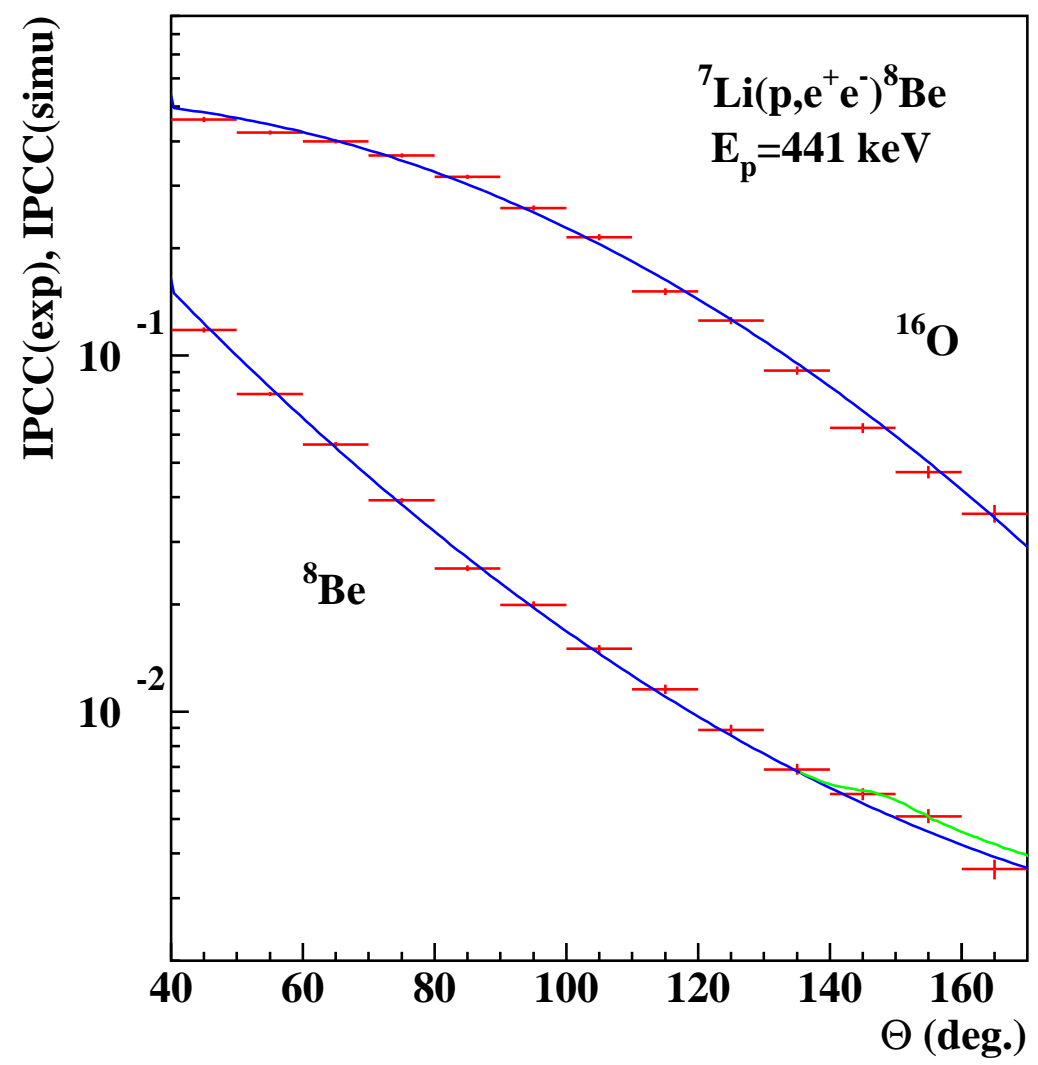

Figure 4: Measured angular correlation of the $e^{+} e^{-}$pairs originated from the decay of the $17.6 \mathrm{MeV}$ resonance compared with the simulated angular correlations [24] assuming M1+1.0\%E1 mixed transitions (full blue curve). The simulated contribution of a $16.7 \mathrm{MeV}$ boson is shown in green.

The $e^{+} e^{-}$decay of a hypothetical boson with mass of $17 \mathrm{MeV} / \mathrm{c}^{2}$ emitted isotropically from the target has been simulated together also with the normal IPC emission of $e^{+} e^{-}$pairs. We have performed a similar fitting procedure like before [7, 8], and obtained a smaller boson to $\gamma$ branching ratio for the anomaly of $(2 \pm 2) \times 10^{-6}$. The result of the fit is shown in green in Fig. 4. It turned out that it is already in the range of our systematical uncertainties, but about 3 times smaller than the one we obtained for the $18.15 \mathrm{MeV}$ transition, previously.

\section{Conclusion}

We have measured the $e^{+} e^{-}$angular correlations for the M1 transition depopulating the 17.64 $\mathrm{MeV}$ state in ${ }^{8} \mathrm{Be}$, and observed a small peak-like deviation from the predicted IPC showing probable X-boson creation in this decay too. The obtained boson to $\gamma$ branching ratio for the anomaly is: $(2 \pm 2) \times 10^{-6}$. To the best of our knowledge, no nuclear physics related description of such deviations can be made [9]. The branching ratio of the $e^{+} e^{-}$decay of such a boson to the $\gamma$ decay for the $17.6 \mathrm{MeV}$ transition $\left(2 \times 10^{-6}\right)$ is less, than for the $18.15 \mathrm{MeV}$ one $\left(5.8 \times 10^{-6}\right)$, which 
agrees with the prediction of Feng et al. [5], and also with the prediction of Kozaczuk, Morrissey and Stroberg [10].

\section{Future plans}

As a next step of the project, we plan to check the creation of the X-boson in the $0^{-} \rightarrow 0^{+}$ $21.01 \mathrm{MeV}$ transition in ${ }^{4} \mathrm{He}$. The $J^{\pi}=1^{+} \mathrm{X}$ boson can be emitted with $\mathrm{L}=1$ angular momentum in the above transition. As the energy of the transition is considerably larger in this case, the created $17 \mathrm{MeV} \mathrm{X}$ boson would move with much larger speed, so the expected maximum of the correlation angle is much smaller $\left(\Theta \approx 105^{\circ}\right)$. The expected background is considerably smaller, since the internal pair creation is forbidden for that transition.

The wide $(\Gamma=0.84 \mathrm{MeV}) 0^{-}$state will be excited in the ${ }^{3} \mathrm{H}(\mathrm{p}, \gamma)^{4} \mathrm{He}$ reaction. The reaction has a very large and positive $\mathrm{Q}$ value $(\mathrm{Q}=19.814 \mathrm{MeV})$, so the low energy tail of the state ( $40 \%$ of the strength) can be excited with $1.00 \mathrm{MeV}$ protons without creating any background as the threshold energy for the ${ }^{3} \mathrm{H}(\mathrm{p}, \mathrm{n})^{3} \mathrm{He}$ reaction is: $\mathrm{E}_{t h}=1.019 \mathrm{MeV}$. However, at this bombarding energy we excite also the first excited state of ${ }^{4} \mathrm{He}\left(\mathrm{E}_{x}=20.21 \mathrm{MeV}, \Gamma=0.50 \mathrm{MeV}\right)$, which has a $\mathrm{J}^{\pi}$ of $0^{+}$so we are expecting $e^{+} e^{-}$pairs from its E0-decay to the ground state by internal pair creation.

Most recently, Ellwanger and Moretti made another possible explanation of the experimental results through a light pseudoscalar particle [9]. Given the quantum numbers of the ${ }^{8} \mathrm{Be}^{*}$ and ${ }^{8} \mathrm{Be}$ states, the $\mathrm{X}$ boson can indeed be a $J^{\pi}=0^{-}$pseudoscalar particle, if it was emitted with $L=1$ orbital momentum. We plan to study the $\gamma \gamma$-decay of the $17-\mathrm{MeV}$ particle, as well in ${ }^{4} \mathrm{He}$, in order to distinguish between the vector boson and the pseudoscalar boson scenarios. According to the Landau-Yang theorem, the decay of a vector boson by double $\gamma$ emission is forbidden, however the decay of a pseudoscalar one is allowed. The angular correlation of the $\gamma$-rays will be measured by using 15 large (3"x3") $\mathrm{LaBr}_{3}$ detectors. If the X boson with a mass of $17 \mathrm{MeV}$ is created in the decay of the $0^{-}$state, and also decays to two $\gamma$ rays, their angular correlation should peak at an angle of:

$$
\cos (\theta)=1-\frac{m_{x}^{2}}{2 E_{\gamma_{1}} E_{\gamma_{2}}},
$$

where $m_{x}$ is the mass of the $\mathrm{X}$ boson $\left(17 \mathrm{MeV} / c^{2}\right)$ and $E_{\gamma 1}$ and $E_{\gamma 2}$ are the energies of the $\gamma$-rays. In our case this expression gives $\theta=105^{\circ}$ for $\gamma$ rays with equal energies.

\section{Acknowledgements}

This work has been supported by the Hungarian NKFIH Foundation No. K124810, and by the European Community FP7 - Contract ENSAR n 262010.

\section{References}

[1] M. Pospelov, A. Ritz, and M. B. Voloshin, Secluded WIMP dark matter, Phys. Lett. B 662, 53 (2008). 
[2] T. Kitahara and Y. Yamamoto, Protophobic light vector boson as a mediator to the dark sector, Phys. Rev. D 95, 015008 (2017).

[3] A.J. Krasznahorkay et al., Phys. Rev. Lett. 116042501 (2016).

[4] Zhang, Gerald and Miller ArXiv: 1703.04588

[5] J. Feng et al., Phys. Rev. Lett. 117, 071803 (2016).

[6] J. Feng et al., Phys. Rev. D95, 035017 (2017).

[7] A.J. Krasznahorkay et al., EPJ Web of Conferences 137, 08010 (2017).

[8] A.J. Krasznahorkay et al., EPJ Web of Conferences 142, 01019 (2017).

[9] Ellwanger and Moretti, JHEP 11039 (2016).

[10] J. Kozaczuk, D.E. Morrissey, S.R. Strobergar, arXiv:1612.01525 (2016).

[11] Bennett GW, et al. (The g-2 Collab.), Phys. Rev. D73, 072003 (2006) [arXiv:hep-ex 0602035].

[12] Antoine Chapelain on behalf of the Muon g-2 Collaboration, arXiv:1701.02807.

[13] L.B. Chen, Y. Liang, C.F. Qiao, arXiv:1607.03970 [hep-ph]; C.H. Chen, T. Nomura, Phys. Lett. B 763 (2016) 304, arXiv:1608.02311 [hep-ph]; F.C. Correia, S. Fajfer, Phys. Rev. D 94 (2016) 115023, arXiv:1609.00860 [hep- ph].

[14] Yi Liang, Long-Bin Chen, Cong-Feng Qiao, Chinese Physics C 41, 063105 (2017).

[15] ATLAS NOTE ATLAS-CONF-2016-042.

[16] R. Corliss, Nucl. Instr. Meth. https://doi.org/10.1016/j.nima.2016.07.053 (in press).

[17] Araki et al., Phys. Rev. D 95, 055006 (2017).

[18] Long-Bin Chen et al., arXiv:1607.03970v2.

[19] Marin Benito et al., Journal of Physics: Conf. Series 800012031 (2017).

[20] Chian-Shu Chen et al., arXiv:1609.07198v1.

[21] Cheng-Wei Chiang (Physics Letters B 767289 (2017).

[22] NA64-2 collaboration, CERN-SPSC-2017-016/ SPSC-SR-211/ 02/04/2017

[23] D.R. Tilley et al., Nucl. Phys. A745, 155 (2004)

[24] J. Gulyás et al., Nucl. Instr. and Meth. in Phys. Res. A 808, 21 (2016)

[25] D. Zahnow et al., Z. Phys. A 351, 229 (1995)

[26] G.A.Fisher et al., Phys.Rev. C14, 28 (1976) 